\title{
Modelling of kinetic interface sensitive tracers reactive transport in 2D two-phase flow heterogeneous porous media
}

\author{
Alexandru Tatomir ${ }^{1, *}$, Dejian Zhou ${ }^{1}$, Huhao $\mathrm{Gao}^{1}$, Alexandru-Nicolae Dimache ${ }^{2}$, Iulian Iancu ${ }^{2}$, and Martin Sauter \\ ${ }^{1}$ Dept. of Applied Geology, Geoscience Centre of the University of Göttingen, Goldschmidtstr. 3, 37077, Göttingen, Germany \\ ${ }^{2}$ Faculty of Hydrotechnics, Technical University of Civil Engineering Bucharest, Bucharest, Romania
}

\begin{abstract}
Fluid-fluid interfacial area plays an important role for mass- and energy-transfer processes across the interface which is relevant in several hydrogeological and engineering applications, e.g. enhanced oil-gas recovery, $\mathrm{CO}_{2}$ storage in geological formations, unconventional geothermal systems, contaminant removal, etc. Kinetic interface sensitive tracers were designed to determine the size of the interface between two fluids by undergoing hydrolysis at the fluid-fluid interface. This study investigates by means of numerical modelling the influence of heterogeneity on the KIS tracer breakthrough curves in six idealized scenarios (S1-S6). It is an extension of the previous work conducted in "one-dimensional" column experiments by Tatomir et al. (2018) [1]. The changes in interfacial area are created by inclusion of heterogeneities at the Darcy-scale. The results show that KIS tracers can be used in two-dimensional experimental setup and can provide information about the size and dynamic evolution of interfacial area. Therefore, this is a first step for the dimensioning of an experimental flume.
\end{abstract}

\section{Introduction}

Developing real-time monitoring techniques able to capture the movement of fluids in porous media is critically important [2], [3]. In particular, recent interest has focused in quantifying the size of the fluid-fluid interfacial area (FIFA) [1], [4]-[9]. Generally, the FIFA can be determined in controlled laboratory conditions by performing microtomographic experiments on millimeter- or centimeter-size samples [10]-[13]. Another laboratory technique is applying tracer methods [14]-[16]. Usually both the $\mu \mathrm{CT}$ - and tracer experiments are conducted at equilibrium. In contrast to these techniques, KIS tracers were developed by [17] to be applied in dynamic flow in porous media systems. [9] showed for the first time the application of these tracers in a controlled dynamic experiment and proposed a method for the determination of the specific IFA. Both studies use n-octane, a non-aqueous phase liquid (NAPL), which is an analogue for the supercritical $\mathrm{CO}_{2}$.

Numerical models capable to simulate the multiphase flow reactive multi-component transport accounting the FIFA have been developed in [2], [18], [19]. The validation of these numerical models was shown by successfully matching the experimental results of NAPL infiltration in a water saturated column. A column experiment can be regarded as a one-dimensional system therefore, the next step towards the application of KIS tracers at field-scale is the dimensioning and testing in laboratory two-dimensional flumes filled with porous media.

Literature observations about laboratory experiments report that even apparently homogeneous samples give flow patterns which are not consistent with standard models [20], [21]. In this sense, small scale heterogeneities cause the flow to develop channels and fingers Fig. 1.

The aim of the current study is to investigate by means of numerical modelling the non-wetting phase plume development together with the KIS tracer transport in a two-dimensional heterogenous porous media system. At the same time aiming to determine the FIFA from the resulting breakthrough curves. a)

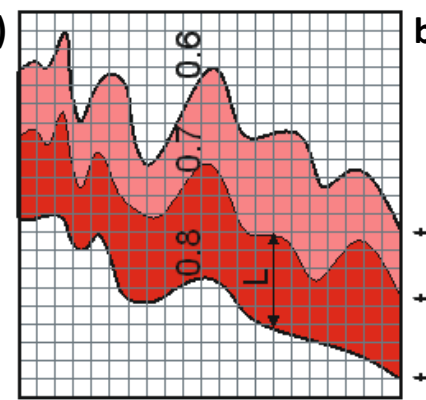

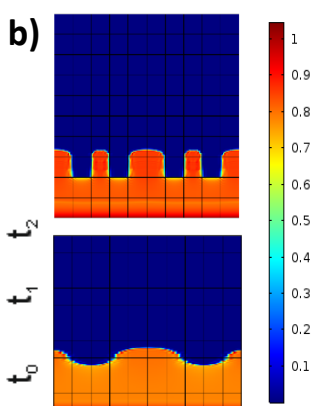

Fig. 1. a) Conceptual representation of the non-wetting phase saturation front spreading with time; b) presence of heterogeneity induces changes in the size of the FIFA.

\section{Mathematical and numerical model}

The immiscible displacement of water from the nonwetting phase can be mathematically formulated using the mass balance eq. (1) using the generalized Darcy's law to express the velocity from eq.(2).

$$
\begin{aligned}
& \frac{\partial\left(S_{\alpha} \phi \rho_{\alpha}\right)}{\partial t}-\nabla \cdot\left(\rho_{\alpha} v_{\alpha}\right)-\rho_{\alpha} q_{\alpha}=0, \\
& \text { with } \alpha=w, n
\end{aligned}
$$

\footnotetext{
* Corresponding author: alexandru.tatomir@geo.uni-goettingen.de
} 


$$
v_{\alpha}=-\mathrm{K} \frac{k_{r \alpha}}{\mu_{\alpha}}\left(\nabla p_{\alpha}-\rho_{\alpha} \mathbf{g}\right) .
$$

where $\alpha$ denotes the phase (with $\mathrm{w}$, as the wetting phase and $\mathrm{n}$ as the non-wetting phase), $S_{\alpha}$ is the phase saturation, $\rho_{\alpha}$ is the phase density, $\phi$ is the porosity of the matrix $q_{\alpha}$ is the phase source or sink term, $\mathrm{K}$ is the intrinsic permeability, $k_{r \alpha}$ is the relative permeability, $\mu_{\alpha}$ is the phase dynamic viscosity, $\mathrm{g}$ is the gravity term, $p_{\alpha}$ is the phase pressure, $p_{c}$ denotes the capillary pressure and $v_{\alpha}$ is the apparent velocity of the fluid as given by the extended multiphase Darcy's law.

The system of two-phase flow partial differential equations (1) is closed with equations (3) and (4):

$$
\begin{aligned}
& S_{w}+S_{n}=1, \\
& p_{n}-p_{w}=p_{c},
\end{aligned}
$$

It can be observed the system does not include the interfacial area. [22] proposed introducing a simplified equation for the balance of specific IFA, i.e., equation (5) which is added to the continuity equations :

$$
\frac{\partial a_{w n}}{\partial t}+\nabla \cdot\left(a_{w n} v_{w n}\right)=E_{w n}
$$

where the interface velocity is expressed:

$$
v_{w n}=-K_{w n} \cdot \nabla a_{w n}
$$

$E_{w n}$ represents the production/destruction rate of specific interfacial area. Even though this term is very important experimental data are still not available for its parametrization. If a porous medium is fully saturated by one phase the IFA is null. Once the displacement process begins the IFA is being created leading to changes in $E_{w n}$. After certain point, the creation of interface stops, and the destruction begins. At this point $E_{w n}=0$. Following this observation, we can formulate:

$$
E_{w n}=-e_{w n} \frac{\partial S_{w}}{\partial t}
$$

where $e_{w n}$ is the strength of the change of $a_{w n}$. And further it leads to :

$$
\frac{\partial a_{w n}}{\partial t}=-e_{w n} \frac{\partial S_{w n}}{\partial t}
$$

Joekar-Niasar et al. (2008) used pore-scale network models to generate computationally $p_{c}-S_{w}-a_{w n}$ surfaces. These can be approximated by using polynomial expression [23], [24]:

$$
\begin{aligned}
a_{w n}\left(S_{w}, p_{c}\right)=a_{0}\left(S_{w}\right)^{a_{1}}(1 & \\
& \left.-S_{w}\right)^{a_{2}}\left(p_{c}^{\max }-p_{c}\right)^{a_{3}}
\end{aligned}
$$

KIS tracers $\left(A_{K I S}\right)$ as defined in [17] are injected dissolved in the non-wetting phase undergo a hydrolysis reaction at the FIFA to produce one acid $\left(B_{\text {acid }}\right)$ and one alcohol $\left(C_{\text {alcohol }}\right)$ :

$$
A_{\text {KIS }}+\mathrm{H}_{2} \mathrm{O} \rightarrow B_{\text {acid }}+C_{\text {alcohol }}
$$

The hydrolysis of esters reaction at the water/NAPL interface commonly is represented by a first-order reaction:

$$
r_{\alpha \rightarrow \beta}^{k}=R_{\alpha \rightarrow \beta}^{k} a_{w n}\left(C_{\beta, s}^{k}-C\right) .
$$

where $R_{\alpha \rightarrow \beta}^{k}$ is the mass transfer coefficient or reaction rate coefficient of component $k$ from phase $\alpha$ to phase $\beta$, $\alpha, \beta$ being the wetting $w$ or non-wetting phase, $n, C_{\beta, S}^{k}$ is the solubility limit of component $k$ in phase $\beta$, and $C_{\beta}^{k}$ is the actual concentration of $k$ in phase $\beta$.

Following the assumptions of [1], [17] the first order reaction in eq. (11) can be simplified to a pseudo-zeroorder kinetic rate as given in eq. (12).

$$
-\frac{d c_{\alpha}^{\kappa}}{d t}=r_{n \rightarrow w}^{\kappa}=R_{c_{n \rightarrow w}{ }^{\kappa}} \cdot a_{w n}
$$

The KIS tracer and its by-products (acid and alcohol) transport in each immiscible phase of the flow phase flow porous medium system can be written:

$$
\begin{gathered}
\frac{\partial\left(\phi S_{\alpha} c_{\alpha}^{\kappa}\right)}{\partial t}-\nabla \cdot\left(c_{\alpha}^{\kappa} \boldsymbol{v}_{\alpha}-D_{p m, \alpha}^{\kappa} \nabla c_{\alpha}^{\kappa}\right) \\
-q_{\alpha}^{\kappa}-r_{\alpha \rightarrow \beta}^{\kappa}=0, \quad \alpha=w, n
\end{gathered}
$$

For the hydrodynamical dispersion tensor we use the formulation of [25]:

$$
\begin{aligned}
D_{p m, \alpha}^{\kappa}= & \delta_{i j} \alpha_{L} v+\left(\alpha_{L}-\alpha_{T}\right) \frac{v_{i} v_{j}}{v} \\
& +\delta_{i j} \phi^{3 / 4} S_{\alpha}^{10 / 3} D_{m}
\end{aligned}
$$

The system of equations (1) - (14) is implemented in the frame of two numerical simulators. The first is the free open-source academic simulator $\mathrm{DuMu}^{\mathrm{x}}$ [26]. The model implementation and numerical results for column experiments are shown in [19]. The second model is a commercial software, COMSOL Multiphysics v5.2 [2]. A benchmarking of the two models is performed in [1]. The results presented in this work were computed only with the Comsol implemented model.

\section{Modelling scenarios}

We investigate six scenarios of an idealized heterogeneous porous medium consisting of a coarse sand with fine sand inclusions (Fig. 2). The ratio of heterogeneous area $(0.4 \mathrm{~m} \times 0.4 \mathrm{~m})$ and surrounding domain is constant in all scenarios except for the first one which is the homogeneous case. The heterogeneities produce deviations of the straight front which induce production and destruction of the FIFA.

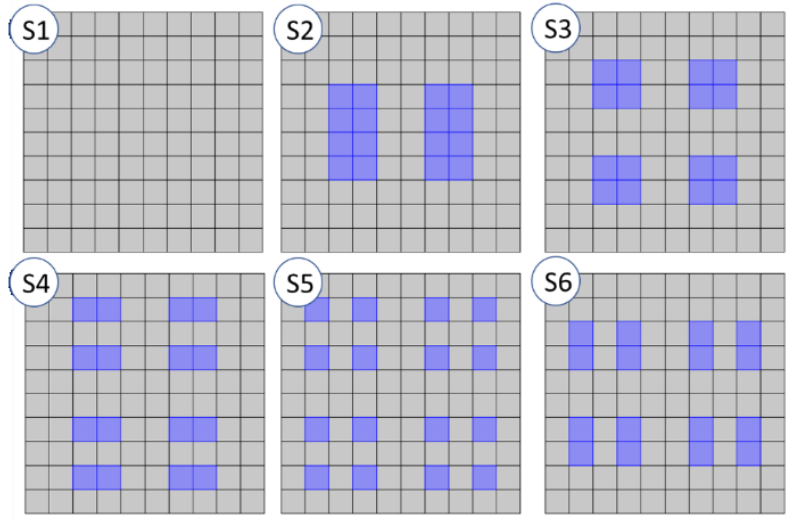

Fig. 2. The six modelling scenarios (S1-S6). S1 is the homogenous case, where the domain is filled only with coarse material. The low permeable inclusions (with higher entry pressure) are highlighted with blue. 
The two-dimensional domain size is $1 \mathrm{~m} \times 1 \mathrm{~m}$. The intrinsic permeability y of the background material is $10 \mathrm{e}-$ $12 \mathrm{~m}^{2}$, while the permeability of the fine material (intrusions) is 100 times lower, i.e., $10 \mathrm{e}-14 \mathrm{~m}^{2}$. The inclusions are represented with a different capillary pressure - saturation - specific IFA relationship than the surrounding porous material.

The entry pressure of the coarse-sand, $p_{d, \text { coarse }}=1700$ $\mathrm{Pa}$, and respectively $p_{d, \text { fine }}=3000 \mathrm{~Pa}$ for the fine-sand. The Brooks-Corey pore index coefficient is for both $\lambda=$ 3 . The coarse-sand entry pressure values are based on the mercury intrusion porosimetry laboratory experiments conducted on glass-beads with the mean dimeter $\mathrm{d}_{50}=240$ $\mu \mathrm{m}$ [9]. Evidently, a finer sand, from the same material will have a higher entry pressure value.

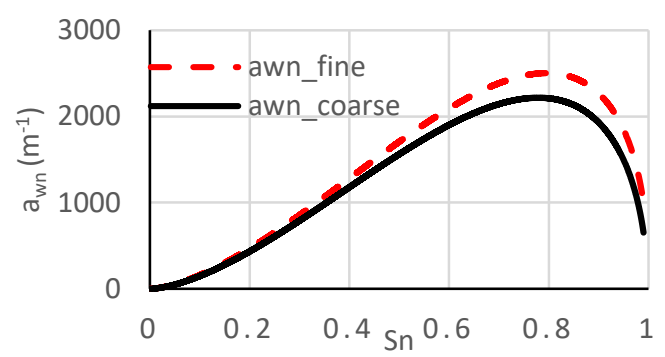

Fig. 3. Specific FIFA - non-wetting saturation relationship.

The coefficients in equation (9) are $a_{0}=2.0327$, $a_{1}=0.41, a_{2}=1.627, a_{3}=0.834$ which are plotted in Fig. 3.

From the dynamic column experiments conducted the specific injection rate of the NAPL is $0.0482 \mathrm{~kg} / \mathrm{s} . \mathrm{m}$ which is maintained over the entire simulation time (10000 s). Fluid prop are maintained constant, i.e. water density $\rho_{w}=1000 \mathrm{~kg} / \mathrm{m}^{3}$, and dynamic viscosity $\mu_{w}=$ $10^{-3} \mathrm{~Pa} \cdot \mathrm{s}, \rho_{N A P L}=703 \mathrm{~kg} / \mathrm{m}^{3}, \quad \mu_{N A P L}=5.42$. $10^{-4} \mathrm{~Pa} \cdot \mathrm{s}$.

\section{Results}

Fig. 4 illustrates the non-wetting phase saturation breakthrough curves at locations $(0.3,0.95),(0.4,0.95)$, $(0.5,0.95)$ and integrated over line $y=0.95$.
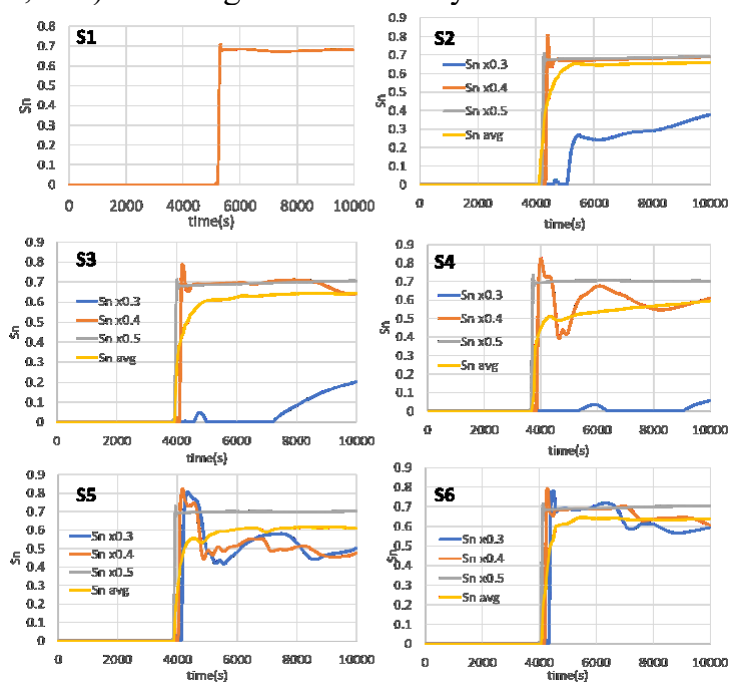

Fig. 4. Non-wetting phase saturation from the six scenarios of investigation at observation points $\mathrm{y}=0.95 \mathrm{~m}$ and $\mathrm{x}=0.3 \mathrm{~m}$, $\mathrm{x}=0.4 \mathrm{~m}, \mathrm{x}=0.5 \mathrm{~m}$ and integrated across the $\mathrm{y}=0.95$ line ( $\mathrm{Sn}$ avg).
The non-wetting saturation at $\mathrm{x} 0.3(0.3,0.95)$ has the highest variability from the four BTC in particular for S2, $\mathrm{S} 3$ and $\mathrm{S} 4$. This is because the position of the observation point is directly above the lower permeable intrusion on the direction of displacement. In all cases NAPL arrival time is faster than in the homogeneous case S1. Little influence is seen at observation point $\mathrm{x} 0.5$, where $\mathrm{Sn}$ remains at $70 \%$.

For the determination of the specific interfacial areas the acid concentration BTCs are being plotted in, Fig. 5. The BTCs show a linear increase with time except at locations $\mathrm{x} 03$ and $\mathrm{x} 04$, where the fluctuation of the nonwetting saturation leads to a fluctuation in specific FIFA and implicitly of the concentration.

For the interpretation of the BTC results we propose the following relationship:

$$
c_{\text {acid }}=a_{w n} \cdot R_{c_{n \rightarrow w}}^{\kappa} \cdot t
$$

Note that the concentration is assumed to have a linear relationship with time and specific FIFA. Table 1 summarized the observed changes in $a_{w n}$ in the six scenarios. The highest relative change is observed for scenarios S4, S3 and S6 which indicates a relationship of the size of heterogeneity relative to the direction of flow.

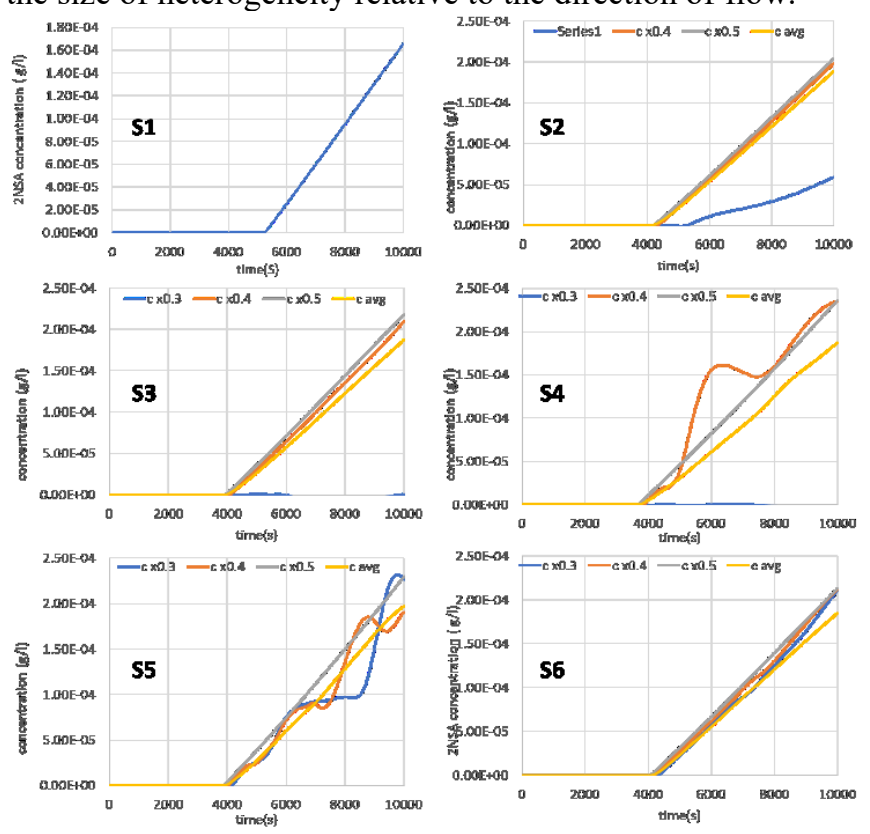

Fig. 5. KIS tracer reaction product acid BTCs at locations $\mathrm{x} 0.3, \mathrm{x} 0.4$, and $\mathrm{x} 0.5, \mathrm{y}=0.95 \mathrm{~m}$.

Table 1. Relative change in interfacial area.

\begin{tabular}{|c|c|c|}
\hline Scenario & FIFA awn $\left(\mathbf{m}^{\mathbf{2}} / \mathbf{m}^{\mathbf{3}}\right)$ & awn increase rate \\
\hline S1 & 2496.49 & $0.00 \%$ \\
\hline S2 & 2368.88 & $-5.11 \%$ \\
\hline S3 & 2298.34 & $-7.94 \%$ \\
\hline S4 & 2256.36 & $-9.62 \%$ \\
\hline S5 & 2459.43 & $-1.48 \%$ \\
\hline S6 & 2314.02 & $-7.31 \%$ \\
\hline
\end{tabular}




\section{Conclusions}

We presented a mathematical model that accounts for immiscible two-phase flow, specific fluid-fluid interfacial area (FIFA) and reactive transport of KIS tracer and reaction products. This research improves the understanding on the production /destruction of the FIFA, for which there is yet no experimental data available to quantify this term. These numerical results are aimed to contribute to the dimensioning of laboratory setups where KIS tracer experiments will be conducted under controlled conditions. Six scenarios (S1-S6) of heterogeneous porous media were investigated to understand the KIS-tracer breakthrough curves and the interfacial area production. Scenario S4 has the highest rate of change in the acid concentration BTC, with a $9.62 \%$ decrease, followed by S3 (-7.94\%) and S6 (7.31\%).

Results show that the location of the observation points leads to differences in the arrival of the non-wetting phase and tracer concentration. Therefore, for the interpretation of the future 2D laboratory BTCs a multiple point sampling system should be considered with the capability to analyse simultaneous samples. Additional modelling studies are required for understanding the importance of the key parameters at macro-scale with the highest influence on the amount of FIFA and its dynamics.

For the future, the design of the field-tracer experiments, will further require multi-disciplinary work of hydrogeologists, engineers, chemist and modellers, as field conditions involve various scales (e.g., presence of fracture, fissures and faults) and types (e.g., mineral heterogeneity, $\mathrm{pH}$ and temperature) of heterogeneities.

This research has received funding by the European Community's 7th Framework Programme FP7 under grant number 309067, TRUST project, and the European Commission Horizon 2020 Research and Innovation Programme under grant agreement 636811, FracRisk project.

\section{References}

[1] A. Tatomir et al., "Kinetic Interface Sensitive Tracers - experimental validation in a two-phase flow column experiment. A proof of concept," Water Resour. Res., vol. DOI: 10.1029/2018WR022621, Oct. 2018.

[2] A. B. Tatomir, A. Jyoti, and M. Sauter, "The Monitoring of $\mathrm{CO} 2$ plume migration in deep saline formations with kinetic interface sensitive tracers," in Geologic Carbon Sequestration: Understanding Reservoir Concepts, T. N. Singh and V. Vikram, Eds. Springer, 2016, p. 336.

[3] A. Niemi, J. Bear, and J. Bensabat, Geological Storage of $\mathrm{CO} 2$ in Deep Saline Formations. Springer, 2017.

[4] S. M. Hassanizadeh and W. G. Gray, "Mechanics and thermodynamics of multiphase flow in porous media including interphase boundaries," Adv. Water Resour., vol. 13, no. 4, pp. 169-186, Dec. 1990.
[5] P. C. Reeves and M. A. Celia, "A Functional Relationship Between Capillary Pressure, Saturation, and Interfacial Area as Revealed by a Pore-Scale Network Model," Water Resour. Res., vol. 32, no. 8, p. 2345, 1996.

[6] V. Joekar-Niasar, S. Hassanizadeh, and A. Leijnse, "Insights into the Relationships Among Capillary Pressure, Saturation, Interfacial Area and Relative Permeability Using Pore-Network Modeling," Transp. Porous Media, vol. 74, no. 2, pp. 201-219, 2008.

[7] M. L. Porter, D. Wildenschild, G. Grant, and J. I. Gerhard, "Measurement and prediction of the relationship between capillary pressure, saturation, and interfacial area in a NAPL-water-glass bead system," Water Resour. Res., vol. 46, no. 8, p. W08512, Aug. 2010.

[8] K. McDonald, K. C. Carroll, and M. L. Brusseau, "Comparison of fluid-fluid interfacial areas measured with X-ray microtomography and interfacial partitioning tracer tests for the same samples," Water Resour. Res., vol. 52, no. 7, pp. 5393-5399, Jul. 2016.

[9] Lyu Ying, Brusseau Mark L., El Ouni Asma, Araujo Juliana B., and Su Xiaosi, "The GasAbsorption/Chemical-Reaction Method for Measuring Air-Water Interfacial Area in Natural Porous Media," Water Resour. Res., vol. 53, no. 11, pp. 9519-9527, Nov. 2017.

[10] E. Dalla, M. Hilpert, and C. T. Miller, "Computation of the interfacial area for two-fluid porous medium systems," J. Contam. Hydrol., vol. 56, no. 1-2, pp. 25-48, May 2002.

[11] M. S. Costanza-Robinson and M. L. Brusseau, "Airwater interfacial areas in unsaturated soils: Evaluation of interfacial domains," Water Resour. Res., vol. 38, no. 10, p. 1195, Oct. 2002.

[12] K. A. Culligan, D. Wildenschild, B. S. B. Christensen, W. G. Gray, and M. L. Rivers, "Pore-scale characteristics of multiphase flow in porous media: A comparison of air-water and oil-water experiments," Adv. Water Resour., vol. 29, no. 2, pp. 227-238, Feb. 2006.

[13] D. Wildenschild and A. P. Sheppard, "X-ray imaging and analysis techniques for quantifying porescale structure and processes in subsurface porous medium systems," Adv. Water Resour., vol. 51, pp. $217-$ 246, Jan. 2013.

[14] H. Kim, P. S. C. Rao, and M. D. Annable, "Gaseous Tracer Technique for Estimating Air-Water Interfacial Areas and Interface Mobility," Soil Sci. Soc. Am. J., vol. 63, no. 6, pp. 1554-1560, Nov. 1999.

[15] L. Chen and T. C. G. Kibbey, "Measurement of airwater interfacial area for multiple hysteretic drainage curves in an unsaturated fine sand," Langmuir ACS J. Surf. Colloids, vol. 22, no. 16, pp. 6874-6880, Aug. 2006.

[16] M. S. Costanza-Robinson and E. J. Henry, "Surfactant-induced flow compromises determination of air-water interfacial areas by surfactant miscibledisplacement," Chemosphere, vol. 171, pp. 275-283, Mar. 2017. 
[17] M. Schaffer, F. Maier, T. Licha, and M. Sauter, "A new generation of tracers for the characterization of interfacial areas during supercritical carbon dioxide injections into deep saline aquifers: Kinetic interfacesensitive tracers (KIS tracer)," Int. J. Greenh. Gas Control, vol. 14, pp. 200-208, May 2013.

[18] J. Niessner and S. M. Hassanizadeh, "Nonequilibrium interphase heat and mass transfer during twophase flow in porous media-Theoretical considerations and modeling," Adv. Water Resour., vol. 32, no. 12, pp. 1756-1766, Dec. 2009.

[19] A. B. Tatomir et al., "Novel approach for modeling kinetic interface-sensitive (KIS) tracers with respect to time-dependent interfacial area change for the optimization of supercritical carbon dioxide injection into deep saline aquifers," Int. J. Greenh. Gas Control, vol. 33, pp. 145-153, Feb. 2015.

[20] P. Langlo and M. S. Espedal, "Macrodispersion for two-phase, immiscible flow in porous media," Adv. Water Resour., vol. 17, no. 5, pp. 297-316, Jan. 1994.

[21] V. I. Heiß, I. Neuweiler, S. Ochs, and A. Färber, "Experimental investigation on front morphology for twophase flow in heterogeneous porous media," Water Resour. Res., vol. 47, no. 10, p. W10528, 2011.

[22] J. Niessner and S. M. Hassanizadeh, "A model for two-phase flow in porous media including fluid-fluid interfacial area," Water Resour. Res., vol. 44, p. 10 PP., Aug. 2008.

[23] V. Joekar-Niasar and S. M. Hassanizadeh, "Uniqueness of Specific Interfacial Area-Capillary Pressure-Saturation Relationship Under NonEquilibrium Conditions in Two-Phase Porous Media Flow," Transp. Porous Media, vol. 94, no. 2, pp. 465-486, Sep. 2012.

[24] A. Tatomir, F. Maier, M. Schaffer, T. Licha, and M. Sauter, "Modelling of Kinetic Interface Sensitive Tracers for Two-Phase Systems," in Clean Energy Systems in the Subsurface: Production, Storage and Conversion, M. Z. Hou, H. Xie, and P. Were, Eds. Springer Berlin Heidelberg, 2013, pp. 65-74.

[25] R. Helmig, Multiphase Flow and Transport Processes in the Subsurface: A Contribution to the Modeling of Hydrosystems, 1st ed. Berlin Heidelberg New York: Springer-Verlag, 1997.

[26] B. Flemisch et al., "DuMux: DUNE for multi\{phase, component, scale, physics, ... flow and transport in porous media," Adv. Water Resour., vol. 34, no. 9, pp. 1102-1112, Sep. 2011. 